\section{Nachweis eines androgenen Wirkstoffes im Hypophysenvorderlappen ${ }^{1}$}

Schon längere Zeit ist es bekannt, daß die männliche Gonade und die daraus hergestellten Lipoide, sowie gewisse reine androgene Wirkstoffe ${ }^{2}$ die physiologische Tätigkeit des Hypophysenvorderlappens (H.V.L.) regulierend beeinflussen ${ }^{3}$. Man kann sich nun die Frage stellen, ob im H.V.L. normaler Tiere ein androgener Wirkstoff in nachweisbarer Menge enthalten ist.

$\mathrm{Da}$ die bisher aus Testes, Urin und Nebenniere isolierten und auch die künstlich hergestellten androgenen Wirkstoffe ausnahmslos der Steroid-Reihe angehören, nahmen wir arbeitshypothetisch an, daß auch ein im H.V.L. vielleicht vorhandener androgener Wirkstoff sich im Lipoid-Anteil befinden sollte. Zur Beantwortung der gestellten Frage mußten verhältnismäßig große Mengen der Lipoide aus H.V.L. verarbeitet werden, da die androgenen Wirkstoffe sogar in der männlichen Gonade, also im Gewebe, in dem sie bisher in höchster Konzentration gefunden worden sind, nur in sehr geringer Menge vorkommen. Als Ausgangsmaterial diente ein von den Wilson Labora tories, Chicago, U.S.A., hergestellter Acetonextrakt aus $267 \mathrm{~kg} \mathrm{H}$. V.L., welche von Rindern beider Geschlechter stammten.

ie Lipoide und besonders die Steroide aus H.V.L. sind in neuerer Zeit von R. E. MARkER und E. L. WITTBECKER ${ }^{4}$ eizgehender untersucht worden. Die amerikanischen Autoren haben den Acetonextrakt aus H.V.L. mit Alkalien verseift und im Unverseifbaren keine anderen Steroide als Choiesterin gefunden. Da durch die energische alkalische Verseifung empfindliche Verbindungen, wie zum Beispiel der einzige bisher aus Testes isolierte androgene Wirkstoff, das Testosteron, zerstört werden, wandten wir zur Anreicherung ein schonenderes Verfahren an, bei welchem diealkalischeVerseifung vermieden wurde. Der Acetonextrakt $(0,82 \mathrm{~kg}$ aus $267 \mathrm{~kg} \mathrm{H}$.V.L.) wurde zuerst von sauren Anteilen (Fettsäuren, Phenolen, Phosphatiden usw.) durch Waschen mit verdünnter Kalilauge befreit's. Aus den neutralen Anteilen $(410 \mathrm{~g})$ trennten wir durch Behandlung mit Petroläther vnd Umlösen aus Aceton den größten Teil $(140 \mathrm{~g})$ des Cholesterins ab. Der cholesterinarme Rückstand wurde in der von ScHort und Gen., Jena, hergestellten Apparatur für diskontinı ierliche Molekular-Destillation bei $0,0001 \mathrm{~mm}$ destilliert und das bis $150^{\circ}$ übergehende Destillat $(58,5 \mathrm{~g})$ mehrmals mit GrRARD Reagens $T^{6}$ behandelt.

Im Gegensatz $z u$ den nicht umgesetzten, unwirksamen Anteilen zeigten die mit GiRARD Reagens $T$ abgetrennten Ketone ( $1,4 \mathrm{~g}$, Anreicherung 1:200000) eine bemerkenswerte androgene Wirksamkeit. In $5 \mathrm{mg}$ des Konzentrates, welche etwa $1 \mathrm{~kg} \mathrm{H}$.V.L. entsprachen, waren 0,5 H.K.E. ?, enthalten. Zum Vergleich sei erwähnt, daß die Stiertestes-Extrakte eine androgeno Wirksamkeit yon etwa 7-20 H.K.E. pro kg Drüse besitzen und daß der Stierharn weniger als $1 \mathrm{H}$.K.E.

1 g. Mitt. Untersuchungen über Organextrakte; 8. Mitt. vg!, Helv, chim. acta 28 im Druck.

2 Als androgene Wirkstoffe bezeichnen wir solche Verbindungen, welche im Kapaunenkamm-Test wirksam sind.

3 Vgl. z. B. F. C. Koch, Physiological Reviews 17, 203 (1937)

4 Journ. Am. Chem. Soc. 63, 1031 (1941).

5 Die Operation wurde zur Vermeidung derOxydation der empfindlichen Inhaltsst offe durch Luftsauerstoffin reinem Stickst off durchgeführt.

6. Girard und G. Sandulesco, Helv.chim. acta 19,1095 (1936).

71 internationale $H, K . E$. (Hahnenkanm-Einheit) $=100 y$ Androsteron $=15 y$ Testosteron.

8 Für die Durchiuhrung der biologischen Auswertung, welche nach dem Verfahren von FussGäNGER durchgeführt wurde, danken wir der biologischen Abteilung der CIBA A.G. in Basel. pro Liter enthält. Da über die Konzentration der androgenen Wirkstoffe im Blut und in anderen Organen nur unzuverlässige Angaben vorliegen ', kann man nicht entscheiden, ob sie im H.V.L, in größerer Menge als in anderen Körperteilen vorkommen.

Die Versuche zur Isolierung des androgenen Wirkstoffes aus H.V.L. in reinem Zustand fihrten bisher nicht zum Erfolg; es gelang jedoch eine Anreicherung.

Durch chromatographische Analyse an Aluminiumoxyd (Aktivität II--IIJ ${ }^{2}$ ) wurden aus der Ketonfraktion ungefähr $3 \%$ an unwirksamem $\Delta^{4}$-Cholestenon-(3) abgetrennt: Smp. $80-81^{\circ}, \quad[\alpha]_{\mathrm{D}}^{25}=+81^{\circ}$. Absorptionsmaximum bei $244 \mathrm{~m} \mu, \log \varepsilon=4,25$ (in Alkohol), o-Tolylsemicarbazon Smp. 230-2310. Wie bei anderen ähnlichen Cholesterin-Umwandlungsprodukten ${ }^{3}$ kann man auch hier nicht entscheiden, ob es sich um ein genuines oder um ein aus Cholesterin während der Verarbeitung entstandenes Produkt handelt.

Die Anreicherung des androgenen Wirkstoffs gelang durch Behandlung der restlichen öligen Ketone mit Digitonin und nochmalige chromatographische Analyse der mit Digitonin nicht gefallten Anteile an Aluminiumoxyd (Aktivität II-III). Nach der Abtrennung der mit Benzol eluierbaren Fraktion, befanden sich die Androgene in den mit $\mathrm{Ather}$ eluierten Anteilen. In 1,5 mg des auf diese Weise erhaltenen Konzentrates war 1 H. K. E. enthalten. Erfahrungsgemä $B$ werden unter den angewandten Versuchsbedingungen solche Steroide mit Ather eluiert, die wenigstens eine Hydroxyl-Gruppe besitzen.

Der angereicherte androgene Wirkstoff war empfindlich gegenüber Alkalien. Durch 4stündiges Kochen mit 3,5proz. methanolischer Kalilauge ging die Wirksamkeit des Konzentrates auf die Hälfte zurück ${ }^{4}$.

Zusammenfassend läBt sich der von uns im H.V.L. nachgewiesene androgene Wirkstoff als ein lipoidlösliches, mit Digitonin nicht fällbares Keton oder ein Gemisch von Ketonen charakterisieren. Diese Eigenschaften sowie das Verhalten beim Chromatographieren und die Empfindlichkeit gegenüber Alkalien weisen auf eine Ahnlichkeit oder sogar Identität mit dem androgenen Wirkstoff aus Testes, dem Testosteron, hin.

Die Versuche zur Isolierung des reinen Wirkstoffes werden fortgesetzt.

Aus dem Rückstand der Molekular-Destillation $(132,5 \mathrm{~g})$, welcher hauptsächlich aus Fetten und Cholesterin-estern bestand, wurden nach der Verseifung mit metha nolischer Kalilauge 42,3g unverseifbare Anteile erhalten. Jaraus lie $\beta$ sich auf dem frither beschriebenen Wege ${ }^{5,6}$ neben Cholesterin analysenteiner Chimylalkohol ( $d-\alpha$-Hexadecyl-glyceryl-äther) isolieren: Smp. $63,5-64^{\circ}$, $[\alpha]_{\mathrm{D}}^{16}=+3,7^{\circ}\left( \pm 0,5^{0}\right)(c=2,0$ in Chloroform), (Diphenylurethan) Smp. 98-98, 50. Der H.V.L. ist das dritte Säugetierorgan, in welchem der Chimyl-alkoholgefunden wurde.

Der Rockefelier Foundation in New York und der CIBA A.G. in Basel danken wir für die Unterstützung.

\section{Prelog und H. C. Beyerman}

Organisch-chemisches Laboratorium der Eidg, Technischen Hochschule, Zürich, den 24. April 1945.

1 Vgl, darüber F. C. Косн, Physiological Reviews 17, 155 (1937).

2 H. Brockmann und H. Schodder, Ber, deutsch. chem. Gesell. 74,73 (1941).

a Vgl darüber V. Prelog, L. Ruzicka und P. Stein, Helv. chim. acta $26,2224(1943)$.

4 Uber die Empfindlichkeit der natürlichen androgenen Wirkstoffe gegenüber Alkalien vil. T. F. Gallaghfr und F. C. КосH, Journ. Biol Chem. 104, 611 (1934).

b. Prelog, L. Ruzicka und F. Stenduann, Helv chim acta 27 , $674(1944)$.

- V. Prelocund H. C. Beyerman, Helv. chimt. acta 28 , 350(1945). 